\title{
Quantitative Electroencephalography as a Diagnostic Tool for Alzheimer's Dementia in Adults with Down Syndrome
}

\author{
Lise Cronberg Salem ${ }^{a} \quad$ Anne Sabers $^{b}$ Troels W. Kjaer ${ }^{\mathrm{e}}$ \\ Christian Musaeus $^{a}$ Martin N. Nielsen ${ }^{c}$ Anne-Grete Nielsen ${ }^{d}$ \\ Gunhild Waldemar ${ }^{a}$ \\ ${ }^{a}$ Danish Dementia Research Centre and ${ }^{b}$ Epilepsy Clinic, Department of Neurology, and \\ 'Department of Clinical Neurophysiology, Copenhagen University Hospital, Rigshospitalet, \\ and ${ }^{\mathrm{d} C e n t r e ~ f o r ~ S e n i o r s ~ w i t h ~ D i s a b i l i t i e s, ~ C o p e n h a g e n, ~ a n d ~}{ }^{\mathrm{e}}$ Neurophysiology Center, \\ Roskilde University Hospital, Roskilde, Denmark
}

\author{
Key Words \\ Down syndrome · Diagnostic assessment · Quantitative electroencephalography · \\ Alzheimer's dementia
}

\begin{abstract}
Background: Assessment of dementia in individuals with intellectual disability is complex due to great inter-individual variability in cognitive function prior to dementia and a lack of standardized instruments. Studies have indicated that quantitative electroencephalography (qEEG) results may be used as a diagnostic marker for dementia. The aim of this study was to examine the value of $\mathrm{qEEG}$ in the diagnostic evaluation of dementia in patients with Down syndrome (DS). Method: The study included 21 patients with DS and mild-to-moderate dementia due to Alzheimer's disease (DS-AD) and 16 age-matched adults with DS without cognitive deterioration assessed by the informant-based Dementia Screening Questionnaire in Intellectual Disability (DSQIID). Conventional EEG was performed and analysed quantitatively using fast Fourier transformation. Outcomes were centroid frequency, peak frequency, absolute power, and relative power. Results: In several regions of the brain, a significant decrease in the theta-1 band $(4-7 \mathrm{~Hz})$ was identified for the centroid frequency. A significant negative correlation was demonstrated between the mean of the centroid frequency of the theta- 1 band and the total DSQIID score. Conclusion: We found that qEEG can detect a significant decrease in centroid frequency in a sample of patients with DS-AD as compared to a sample of adults with DS and no cognitive deterioration.

(C) 2015 S. Karger AG, Basel
\end{abstract}




\section{Introduction}

In developed countries, the population of elderly people with Down syndrome (DS) is expanding, resulting in an increasing incidence of age-related diseases including dementia $[1,2]$. DS is the most common chromosomal defect leading to mental retardation, with an incidence of approximately 1 in 650-1,000 live births worldwide [1]. It is caused by trisomy of chromosome 21 and is associated with physical, psychiatric, and neurological complications. Neuritic amyloid- $\beta$ plaques and neurofibrillary tangles - characteristic neuropathological features of Alzheimer's disease (AD) - are a virtually certain finding in the brains of adults with DS, and have even been noted in some children with DS [3-6]. The earliest accumulation appears to be in the frontal and temporal lobes [6]. The increased risk of AD in DS is primarily attributed to a gene found on chromosome 21, amyloid precursor protein, although the trigger for development of dementia is unknown [7]. It is estimated that, clinically, dementia can be diagnosed in only some $9 \%$ of adults with DS in their 4 th decade and around 55\% in their 6th decade of life [8]. Another study has estimated that nearly $20 \%$ of individuals with DS aged 45 years and more have dementia due to AD (DS-AD), and several studies have shown a greater risk of early mortality for people with DS and dementia [6, 9-12]. However, the incidence rate of dementia is associated with uncertainty, as the diagnosis of dementia in people with DS remains difficult, particularly in the early stage of the disease.

The assessment of dementia in individuals with intellectual disability is often complex due to great inter-individual variability in cognitive functioning prior to dementia and a lack of standardized measures to detect dementia. The ability to cooperate in relation to the successful completion of standard physical examinations, brain imaging, and other investigatory tests is often impaired due to intellectual disability $[13,14]$. The diagnostic assessment may be further complicated due to the fact that social and cognitive abilities often are underdeveloped and comorbidity of hypothyroidism and depression is relatively common [13]. To circumvent these problems, it is recommended that neuropsychological testing of a patient should be combined with informant-based rating scales.

Quantitative electroencephalography (qEEG) is the analysis of various features of conventional EEGs to derive metrics and visual displays through digital methods. The electrical activity is quantified with a variety of statistical measures to characterize the huge variation in combinations of emissions from the brain. Studies have shown that AD leads to increasing slow-wave activity of the EEG (an increase in theta and delta wave activity) and enhanced complexity of the EEG signals [15]. In adults with DS without dementia, it has been established that an age-related slowing of the alpha rhythm may develop at an earlier age as compared to healthy adults of the general population [16]. However, to our knowledge there are no reports comparing qEEG results in patients with DS with and without AD. It is possible that EEG, an atraumatic investigation, may contribute to the early diagnosis of AD in DS.

The aim of the present study was to examine the value of qEEG in the diagnostic evaluation of dementia in patients with DS. We hypothesized an increase in delta and theta wave activity of the qEEG recordings in patients with DS and AD globally as compared to people with DS without AD.

\section{Subjects and Methods}

This study was designed as an explorative case-control study on qEEG as a diagnostic tool in patients with $\mathrm{DS}$ and possible dementia due to $\mathrm{AD}$. 


\section{Sample}

Patients with DS and cognitive decline were recruited from the Copenhagen University Hospital Memory Clinic at Rigshospitalet, which is a multidisciplinary outpatient clinic based in the Department of Neurology. Referrals are received from general practitioners, specialists, and other hospitals.

The control subjects were adults with DS recruited from recreational homes for adults with intellectual disabilities in the Copenhagen area. An administrative specialized nurse supervising the institutions contacted the manager of each institution, asking for permission to contact potential participants according to the inclusion criteria applied for this study.

The inclusion criteria for both patients and control subjects were: (1) karyotype examination confirming trisomy of chromosome 21; (2) ability to cooperate (leading to exclusion of participants with severe intellectual disability); (3) age above 35 years, and (4) fulfilling the criteria for probable AD. The exclusion criterion for patients and control subjects was a mis- or under-treated medical (assessed by blood tests and clinical appearance) or psychiatric condition (assessed clinically by the neurologist) that might influence cognition (e.g. hypothyroidism and depression).

We recruited 21 patients with DS and AD and 16 age-matched control subjects with DS and no cognitive decline. Cognitive decline was assessed using the informant-based Dementia Screening Questionnaire in Intellectual Disability (DSQIID) [17]. One patient and 2 control subjects were living at home, attending a supervised workshop, while the remaining patients and control subjects were institutionalized.

Informed consent was obtained from the personal legal guardian, or, if no legal guardian was assigned, from the family doctor. The subjects and caregivers were informed that they could request the interruption of the clinical and instrumental procedures at any time.

\section{Diagnostic Workup of the Patients}

The dementia diagnosis was established based on the following assessments:

1 A detailed history of medical, neurological, and psychiatric conditions, including a review of the medical records, to establish past and present physical and/or mental health disorders

2 A history of symptoms of dementia from family members and/or caregivers

3 Information on the current intake of medicine

4 Screening for dementia symptoms using the DSQIID [17], an informant-based questionnaire; a high score $(>20)$ indicates a high level of cognitive decline; this screening instrument has been proved to be a valid and reliable observer-rated questionnaire for screening for dementia among adults with DS

5 Physical and neurological examinations including a comprehensive screening for visual and hearing impairment

6 Psychiatric assessment of symptoms of depression (when relevant)

7 A full panel of blood tests recommended as part of the diagnostic workup of dementia according to international clinical guidelines available in 2013 [18]

8 Confirmation of genetic status (trisomy 21) from the medical records

9 CT scanning of the brain if the patient was able to cooperate

10 Additional examinations (when found relevant)

Subsequently, the diagnosis of dementia was established according to the ICD-10 [19] and/ or DSM-IV criteria [20]. The diagnosis of AD was established according to the criteria of the National Institute of Neurological and Communicative Disorders and Stroke and the Alzheimer's Disease and Related Disorders Association (NINCDS-ADRDA) for probable AD [21], supported by the ICD-10 Symptom Checklist for Mental Disorders and guidelines set by an international consensus panel associated with the International Association for the Scientific Study of Intel- 
lectual Disabilities (IASSID) [22]. After completion of the diagnostic workup, the multidisciplinary staff (dementia specialists, neuropsychologists, and specialist nurses) established a consensus diagnostic classification. The patients with DS-AD included in this study were estimated to be in a mild-to-moderate phase of their dementia disorder. Each control subject with DS included in this study was examined at baseline according to items 1-8.

\section{Electroencephalography}

Electroencephalograms were recorded using Nicolet One EEG (Nervus) recording software 5.82 (Natus) with a standard 44-channel head box, and each subject was fitted with a cap using silver-silver-chloride-coated electrodes. The electroencephalogram was recorded in a 30-min period in subjects in the wake resting state from 19 electrodes positioned according to the International 10-20 system (i.e. Fp1, Fp2, F3, Fz, F4, F7, F8, T3, T4, C3, Cz, C4, T5, T6, P3, Pz, P4, 01, 02). Data were sampled at $1.0 \mathrm{kHz}$ and bandpass filtered at 0.5-70.0 $\mathrm{Hz}$. To monitor eye movements and heart rhythm, electrooculograms and electrocardiograms (0.3-70 Hz bandpass) were collected. The status of handedness for every participant was established using the Edinburgh inventory [23].

Special attention was devoted to obtaining maximal relaxation and cooperation of the subjects, to obtaining high-quality EEG recordings, and to avoiding well-known effects of stress and anxiety. An experienced neurophysiologist (T.W.K.) analysed the conventional electroencephalograms blindly, unaware of the clinical data on the subjects. The recorded EEG data were then fragmented off-line in consecutive epochs of a minimum of $4 \mathrm{~s}$ by a medical student (C.M.), also blinded to the clinical data on the patients. For each subject, up to 10 epochs were carefully selected for analysis. However, in some cases the subjects were unable to lie still, resulting in artefacts on EEG and less than 10 epochs suitable for analysis. None of the EEG recordings revealed paroxysmal activity. Seven frequency bands were determined for analysis: delta $(0.5-3.5 \mathrm{~Hz})$, theta- $1(4-7 \mathrm{~Hz})$, theta-2 $(5-6 \mathrm{~Hz})$, theta-3 $(5-7 \mathrm{~Hz})$, theta-alpha-1 (5-10 Hz), theta-alpha-2 (4-13 Hz), alpha (8-13 Hz), and beta ( $\geq 14 \mathrm{~Hz})$.

\section{Quantitative EEG}

qEEG spectral analysis was performed using digital fast Fourier transformation and power spectral density (PSD) determination with the Welch method (Hamming window function, 75\% overlap, and 4-second window length). Four qEEG parameters were derived from the PSD: centroid frequency, peak frequency, absolute power, and relative power. An illustration of the qEEG outcome is shown in figure 1. Determined by PSD, centroid frequency was defined as the weighted mean of the frequencies present in the EEG epoch, using their magnitudes as weights. Peak frequency was defined as the peak frequency in the respective epoch, channel, and frequency band with highest magnitude in the PSD. Absolute power was defined as the total power in each epoch, channel, and frequency band normalized to $1 \mathrm{~s}$. Relative power was defined as the relative power in each frequency band compared to the total power in all frequency bands, in each epoch and channel.

All qEEG parameters were computed for each epoch, for all channels, and in all selected frequency bands using a MATLAB-based GUI program developed for this study.

\section{Ethics}

This study was approved by the local ethics committee (J.nr. H-4-2012-111) and the Danish Data Protection Agency (J.nr. 2007-58-0015).

\section{Statistical Analysis}

The level of significance in group-by-group comparisons was investigated with the independent sample $t$ test for quantitative variables with a normal distribution. The Mann- 


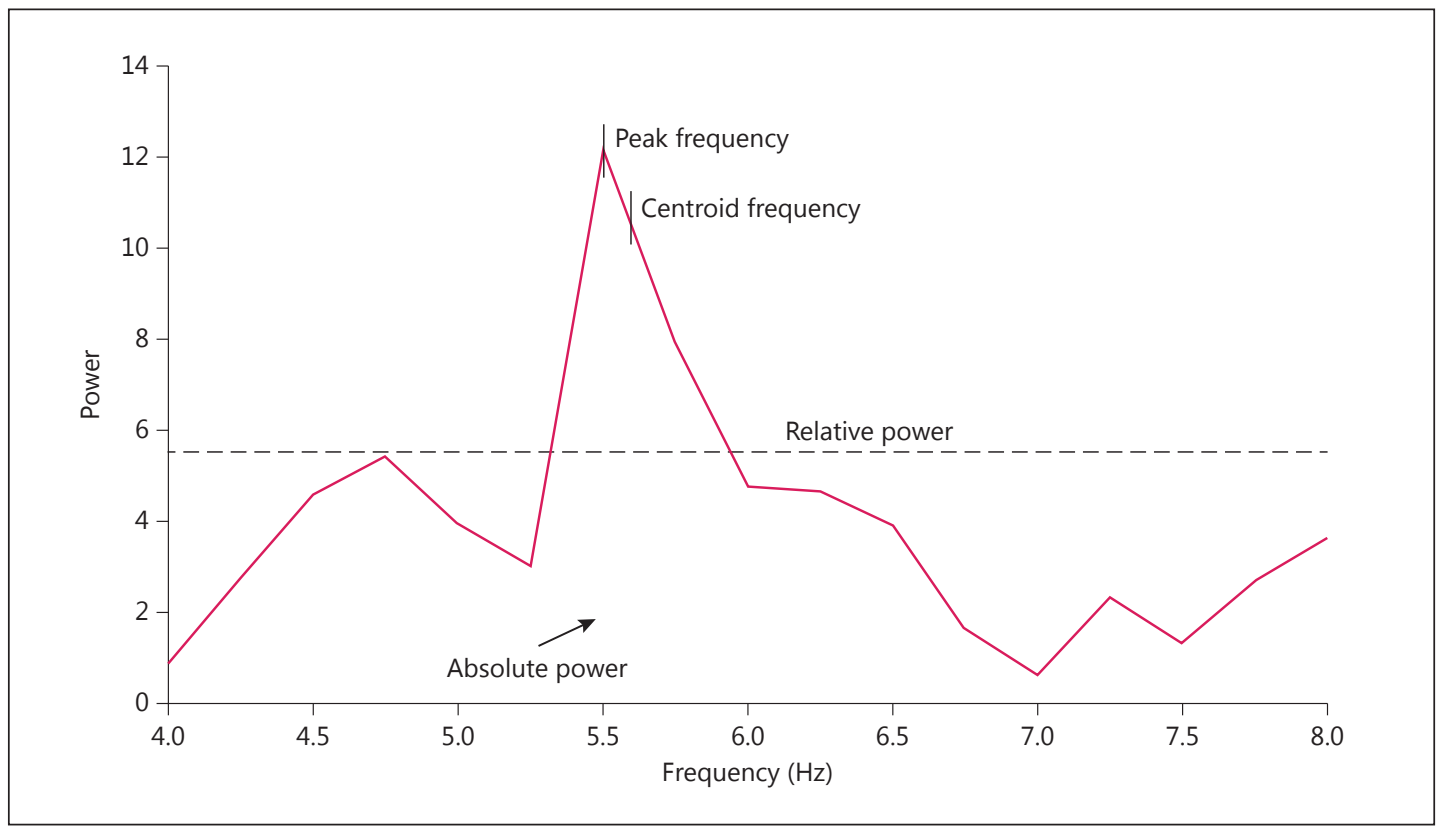

Fig. 1. Illustration of the outcome of the qEEG analysis.

Table 1. Clinical data on the patients and controls with DS

\begin{tabular}{llll}
\hline & $\begin{array}{l}\text { Patients } \\
\text { (DS-AD) }\end{array}$ & $\begin{array}{l}\text { Controls } \\
\text { (DS) }\end{array}$ & $\mathrm{p}$ value \\
\hline Subjects, $\mathrm{n}(\%)$ & $21(57)$ & $16(43)$ & \\
Mean age (range), years & $52.5(40-63)$ & $48.5(38-68)$ & $\mathrm{p}=0.099^{1}$ \\
Male-female ratio, $\mathrm{n}$ & $18: 3$ & $16: 10$ & $\mathrm{p}=0.103^{2}$ \\
Handedness (L/R), $\mathrm{n}$ & $2 / 19$ & $2 / 14$ & $\mathrm{p}<0.001^{3}$ \\
Mean DSQIID score \pm SD & $21.7 \pm 6.2$ & $2.8 \pm 3.0$ & \\
\hline${ }^{1}$ t test. ${ }^{2} \chi^{2}$ test. ${ }^{3}$ Mann-Whitney U test. & \\
\hline
\end{tabular}

Whitney U test was used for group-by-group comparisons with a skewed distribution of the data. The $\chi^{2}$ test was used on nominal variables, and Fischer's exact test (two-sided) was applied when cells had expected counts of less than 5 . A linear regression model was used to assess the impact of centroid frequency, peak frequency, absolute power, and relative power in the theta- 1 band on DSQIID score, with plots of residuals as a model control. The false discovery rate was used to correct for multiple comparisons. The data were analysed using SPSS 19.0 for Windows (SPSS Inc., Chicago, Ill., USA).

\section{Results}

\section{Clinical Data}

The clinical data are shown in table 1 . As expected, total scores on the DSQIID were significantly different between patients and control subjects $(U=0.0, z=-5.17, p<0.001)$, as the highest score was found in the DS-AD group. No significant group differences were found in age and sex ratios between patients and control subjects. 
Salem et al.: Quantitative Electroencephalography as a Diagnostic Tool for Alzheimer's

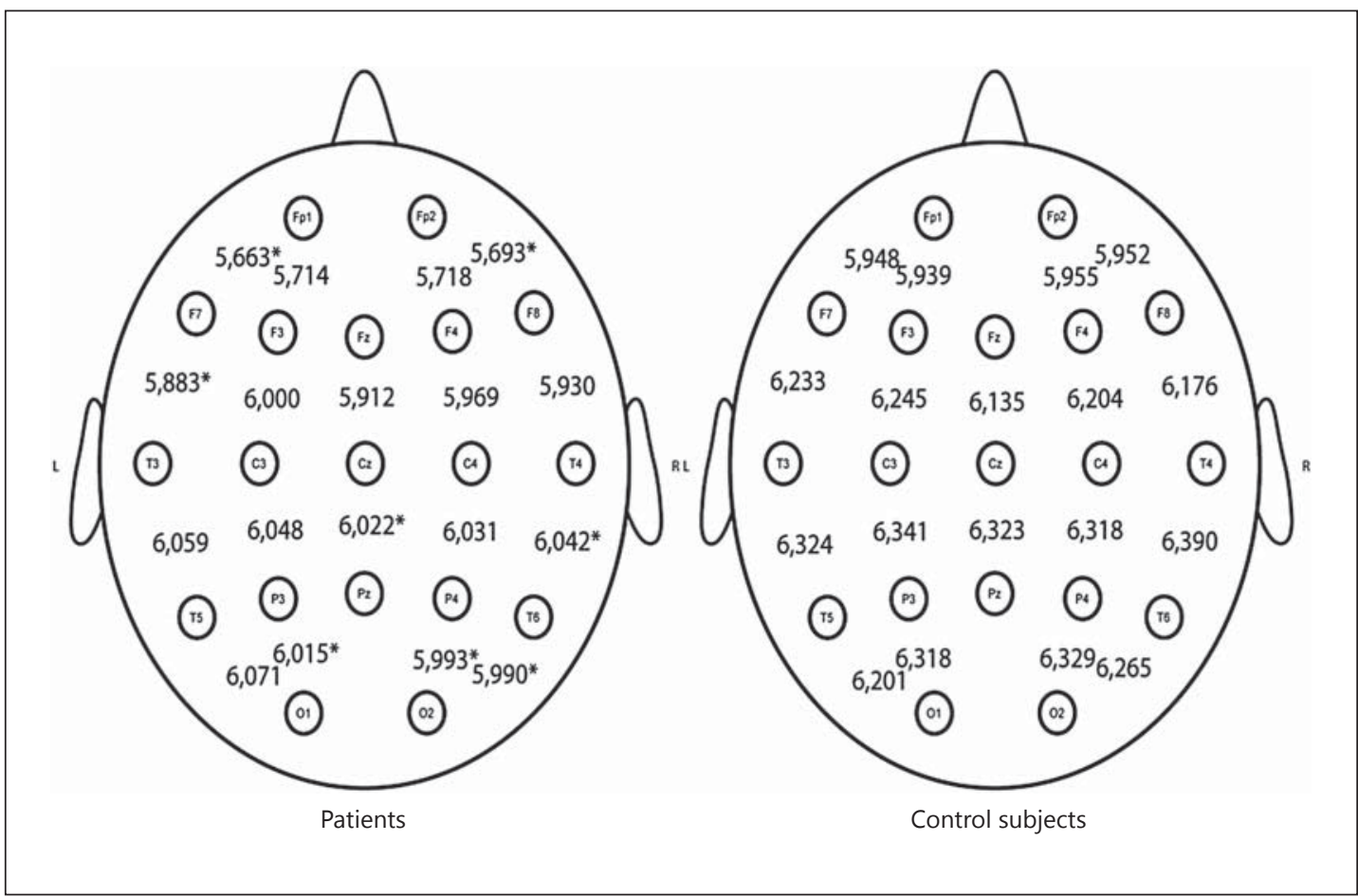

Fig. 2. Mean scores $(\mathrm{Hz})$ of centroid frequency in the theta-1 band for all the regions investigated in patients and control subjects. A decrease in centroid frequency is evident globally in patients as compared to control subjects and significantly different in several areas of the brain (frontotemporal on both sides and parietal on the left side). * Significantly different from control subjects at $\mathrm{p}<0.05$ ( $\mathrm{t}$ test).

\section{Mean Scores of Centroid Frequency Globally for Every Frequency Band for the}

Two Study Groups

Mean scores of centroid frequency, mean frequency, absolute power, and relative power of every frequency band in patients were compared to those in control subjects. The centroid frequency for the theta- 1 band was significantly decreased in several areas of the brain primarily frontotemporal, on the left side - in patients with DS-AD as compared to control subjects (fig. 2). This was specifically the case in the theta-1 band for Fp1-F7 [ $(\mathrm{t}=35)=2.681$, $\mathrm{p}=0.001]$ and $\mathrm{F} 7-\mathrm{T} 3(\mathrm{U}=82, \mathrm{z}=-2.64, \mathrm{p}=0.008, \mathrm{r}=0.43)$. The false discovery rate was used to correct for multiple comparisons leading to insignificant $\mathrm{p}$ values for all frequency bands. To visualize the negative correlation between the mean of the centroid frequency of the theta-1 band in the left frontotemporal region (F7-T3) and the total score on the DSQIID, a scatterplot was generated (fig. 3).

No significant differences were found for the remaining variables (peak frequency, absolute power, or relative power) globally between patients and control subjects in any of the included frequency bands.

Four patients were left-handed. These patients were assumed right-hemisphere dominant, while the rest were considered left-hemisphere dominant. All data were re-analysed with a dominance laterality covariate. This did not change any conclusions.

Correlation between qEEG Variables Globally and Total Score on the DSQIID

When the mean of the centroid frequency of the theta-1 band at F7-T3 was entered into a stepwise linear regression model of the DSQIID, the total variance explained by the model 
Fig. 3. Means of centroid frequency recorded in the frontotemporal region (F7-T3) on the left side for the theta- 1 band compared to the total score on the DSQIID. A negative correlation is seen between the quantity of symptoms of dementia and the mean of the centroid frequency for the theta-1 band. $\bigcirc=$ Control subjects; $\bigcirc=$ DS-AD subjects.

Fig. 4. Correlation between the mean of the centroid frequency in the theta- 1 band and the total score on the DSQIID using multiple linear regression analysis in all the regions of the brain investigated. Pearson's correlation coefficient is shown in the vertical bar, correlating to a shift in colour. The correlation between a high quantity of symptoms of dementia and a decrease in the centroid frequency for the theta-1 band is evident globally and most pronounced frontotemporal on the left side.

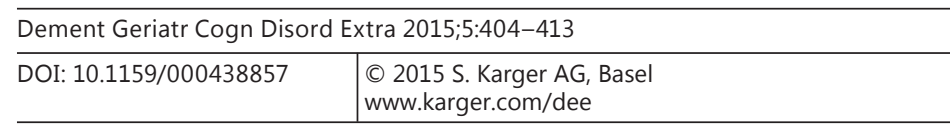

Salem et al.: Quantitative Electroencephalography as a Diagnostic Tool for Alzheimer's Dementia in Adults with Down Syndrome
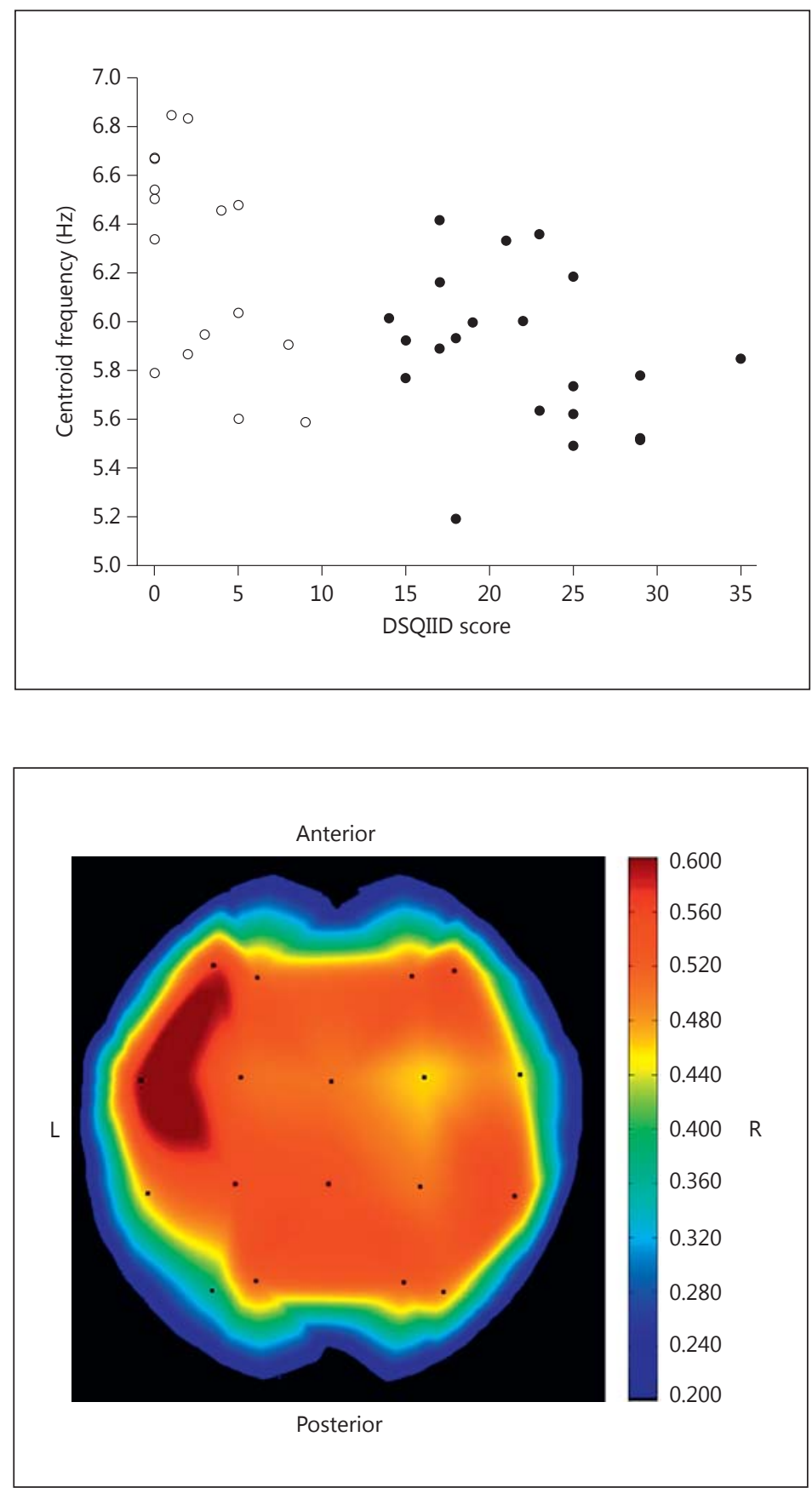

as a whole was $32 \%$ of the variance in DSQIID score $[F(2,34)=7.98, p<0.001]$. Figure 4 shows Pearson's correlation coefficients of the mean of the centroid frequency of the theta-1 band for all the regions investigated, and a shift in colour from blue to red indicates an increasing correlation coefficient. Interestingly, a correlation of $52 \%$ or higher between centroid frequency for the theta- 1 band and the level of symptoms of dementia was seen globally in the brain and most prominent in the left frontotemporal region. These results indicate that increasing cognitive impairment is correlated to a decrease in centroid frequency in the theta- 1 band globally. 


\section{Discussion}

To our knowledge, this is the first study to examine the diagnostic value of qEEG in a clinical setting in patients with DS-AD as compared to adults with DS and no cognitive decline. Our results show a significant decrease in centroid frequency in the theta- 1 band in patients with DS-AD in several regions of the brain (fig. 2). Furthermore, we have demonstrated a significant correlation between high scores on the DSQIID and lower frequency of the qEEG variable centroid frequency in the theta- 1 band globally and, most pronounced, in the left frontotemporal region. These results are unique, but they may be biased by the relatively small study population and by the fact that patients with DS-AD on average were 4 years older than control subjects. As higher age is associated with a higher incidence of symptoms of dementia and slowing of the EEG, this is a confounding factor to be taken into consideration. Furthermore, correcting for multiple comparisons using the false discovery rate resulted in a non-significant decrease in centroid frequency.

The decrease in centroid frequency in the theta- 1 band is in accordance with previous findings showing an increased slow-wave activity of the delta and theta frequency bands in adults with DS-AD as compared to healthy adults [24, 25]. A number of studies have previously demonstrated an association between EEG abnormalities and AD in adults with DS. Visser et al. [25] showed, in a prospective setting, a decrease in frequency of the dominant occipital activity at the onset of cognitive deterioration, in agreement with Soininen et al. [24], who found significantly more slow-wave activity (theta and delta) in patients with DS-AD as compared to healthy control subjects without DS. Both studies used adults without intellectual disabilities as control subjects, and because of this, it cannot be determined whether the results found were due to DS, AD, or a combination of the two. This study used controls with DS and thereby allowed the effect of AD to be better examined. Adults with DS without cognitive deterioration have been shown in several studies to have abnormal EEG results characterized by the absence of a normal alpha rhythm [26,27]. Therefore, in order to be able to separate patients with DS-AD from adults with DS with cognitive impairment due to other causes, we found it preferable in a clinical setting as a first step to compare results of qEEG in patients with DS-AD to those in other adults with DS without cognitive deterioration.

In adults with intellectual disabilities, a large inter-individual variability in cognitive and functional abilities is present, premorbid levels of cognitive functioning are generally unknown, and their ability to cooperate in diagnostic assessments, including cognitive testing, is often impaired, leading to a low validity of these tests $[13,28]$. As a consequence, all participants of this study were screened for dementia symptoms using an informant-based assessment tool, the DSQIID [17]. We found a significant correlation between centroid frequency and total score on the DSQIID, most pronounced in the left frontotemporal region (fig. 3). This correlation may indicate a decreasing frequency of EEG recordings related to cell death in these areas due to accumulating amyloid plaques and neurofibrillary tangles. These findings are related to previous findings on patterns of neuropathological changes in elderly adults with DS [5, 6, 29]. Mann [5] found that these neuropathological findings in adults with DS beyond 40 years of age primarily involved the amygdala, hippocampus, and association areas of the frontal, temporal, and parietal cortices.

As shown in figure 3, there is an overlap between adults with DS with dementia and those without dementia in the $5.5-$ to $6.5-\mathrm{Hz}$ frequency bands. Based on the small sample size, it is not possible to determine a cutoff value. Presumably, larger study population is needed to clarify this issue.

Our results indicating a dominant decrease in centroid frequency on the left side are not supported by previous studies and may be coincidental. Theoretically, they could be related 
Salem et al.: Quantitative Electroencephalography as a Diagnostic Tool for Alzheimer's Dementia in Adults with Down Syndrome

to symptoms of aphasia associated with the left temporal lobe. Aphasia may be a symptom of dementia and may lead patients to diagnostic evaluation [30].

Neurophysiological assessments of brain activity have been undertaken to aid the clinical diagnosis of dementia in adults with intellectual disability due to the poor validity of neuropsychological measures for detecting cognitive deterioration in this population [13]. As conventional EEG recordings are non-invasive, EEG may serve as a potential diagnostic tool and as a predictor of AD suitable for patients with intellectual disabilities. Furthermore, EEG recordings are inexpensive, potentially mobile, and relatively well tolerated. All participants of our study were able to complete a conventional EEG examination.

In conclusion, we found that $\mathrm{qEEG}$ can detect a significant decrease in centroid frequency in a sample of patients with DS-AD as compared to a sample of adults with DS and no cognitive deterioration. This is a unique finding, calling for further elaboration and larger studies in order to define possible cutoff values and clinical implications. Further studies are needed to elaborate these aspects.

\section{Acknowledgements}

The authors would like to thank the Danish Research Foundation for a scholarship to L.C.S. as well as the Danish Health Foundation and the Danish Ministry of Health for support of the Danish Dementia Research Centre.

\section{Disclosure Statement}

The authors have no actual or potential conflicts of interest to declare.

\section{References}

1 Bittles AH, Bower C, Hussain R, Glasson EJ: The four ages of Down syndrome. Eur J Public Health 2007; 17: 221-225.

2 Holland AJ: Ageing and learning disability. Br J Psychiatry 2000;176:26-31.

3 Olson MI, Shaw CM: Presenile dementia and Alzheimer's disease in mongolism. Brain 1969;92:147-156.

-4 Wilcock DM, Griffin WS: Down's syndrome, neuroinflammation, and Alzheimer neuropathogenesis. J Neuroinflammation 2013;10:84.

5 Mann DM: The pathological association between Down syndrome and Alzheimer disease. Mech Ageing Dev 1988;43:99-136.

6 Zigman WB, Lott IT: Alzheimer's disease in Down syndrome: neurobiology and risk. Ment Retard Dev Disabil Res Rev 2007;13:237-246.

-7 Schupf N, Sergievsky GH: Genetic and host factors for dementia in Down’s syndrome. Br J Psychiatry 2002; 180:405-410.

-8 Prasher VP, Filer A: Behavioural disturbance in people with Down's syndrome and dementia. J Intellect Disabil Res 1995;39:432-436.

\9 Coppus AM, Evenhuis HM, Verberne GJ, Visser FE, Oostra BA, Eikelenboom P, van Gool WA, Janssens AC, van Duijn CM: Survival in elderly persons with Down syndrome. J Am Geriatr Soc 2008;56:2311-2316.

10 Prasher VP: Down's syndrome, longevity, and Alzheimer's disease. Br J Psychiatry 1993;162:711.

11 Thase ME: Longevity and mortality in Down's syndrome. J Ment Defic Res 1982;26:177-192.

12 McCarron M, McCallion P, Reilly E, Mulryan N: A prospective 14-year longitudinal follow-up of dementia in persons with Down syndrome. J Intellect Disabil Res 2014;58:61-70.

13 Nieuwenhuis-Mark RE: Diagnosing Alzheimer's dementia in Down syndrome: problems and possible solutions. Res Dev Disabil 2009;30:827-838.

14 Prasher V, Farooq A, Holder R: The Adaptive Behaviour Dementia Questionnaire (ABDQ): screening questionnaire for dementia in Alzheimer's disease in adults with Down syndrome. Res Dev Disabil 2004;25:385-397.

15 Dauwels J, Vialatte F, Cichocki A: Diagnosis of Alzheimer's disease from EEG signals: where are we standing? Curr Alzheimer Res 2010; 7:487-505. 


\begin{tabular}{l|l}
\hline DOI: $10.1159 / 000438857$ & $\begin{array}{l}\text { C } 2015 \text { S. Karger AG, Basel } \\
\text { www.karger.com/dee }\end{array}$ \\
\hline
\end{tabular}

Salem et al.: Quantitative Electroencephalography as a Diagnostic Tool for Alzheimer's Dementia in Adults with Down Syndrome

16 Murata T, Koshino Y, Omori M, Murata I, Nishio M, Horie T, Isaki K: Quantitative EEG study on premature aging in adult Down's syndrome. Biol Psychiatry 1994;35:422-425.

17 Deb S, Hare M, Prior L, Bhaumik S: Dementia screening questionnaire for individuals with intellectual disabilities. Br J Psychiatry 2007;190:440-444.

18 Hort J, O’Brien JT, Gainotti G, Pirttila T, Popescu BO, Rektorova I, Sorbi S, Scheltens P: EFNS guidelines for the diagnosis and management of Alzheimer's disease. Eur J Neurol 2010;17:1236-1248.

19 World Health Organization: The ICD-10 Classification of Mental and Behavioral Disorders: Diagnostic Criteria for Research. Geneva, World Health Organization, 1993.

20 American Psychiatric Association: Diagnostic and Statistical Manual of Mental Disorders. Washington, American Psychiatric Association, 2000.

-21 McKhann G, Drachman D, Folstein M, Katzman R, Price D, Stadlan EM: Clinical diagnosis of Alzheimer's disease: report of the NINCDS-ADRDA Work Group under the auspices of Department of Health and Human Services Task Force on Alzheimer's Disease. Neurology 1984;34:939-944.

22 Aylward EH, Burt DB, Thorpe LU, Lai F, Dalton A: Diagnosis of dementia in individuals with intellectual disability. J Intellect Disabil Res 1997;41:152-164.

23 Oldfield RC: The assessment and analysis of handedness: the Edinburgh inventory. Neuropsychologia 1971; 9:97-113.

24 Soininen H, Partanen J, Jousmäki V, Helkala EL, Vanhanen M, Majuri S, Kaski M, Hartikainen P, Riekkinen P Sr: Age-related cognitive decline and electroencephalogram slowing in Down's syndrome as a model of Alzheimer's disease. Neuroscience 1993;53:57-63.

25 Visser FE, Kuilman M, Oosting J, Overweg J, van Wijk J, van Huffelen AC: Use of electroencephalography to detect Alzheimer's disease in Down's syndrome. Acta Neurol Scand 1996;94:97-103.

26 Evenhuis HM: The natural history of dementia in Down's syndrome. Arch Neurol 1990;47:263-267.

27 Menendez M: Down syndrome, Alzheimer's disease and seizures. Brain Dev 2005;27:246-252.

28 Krinsky-McHale SJ, Silverman W: Dementia and mild cognitive impairment in adults with intellectual disability: issues of diagnosis. Dev Disabil Res Rev 2013;18:31-42.

-29 Azizeh BY, Head E, Ibrahim MA, Torp R, Tenner AJ, Kim RC, Lott IT, Cotman CW: Molecular dating of senile plaques in the brains of individuals with Down syndrome and in aged dogs. Exp Neurol 2000;163:111-122.

-30 Harciarek M, Cosentino S: Language, executive function and social cognition in the diagnosis of frontotemporal dementia syndromes. Int Rev Psychiatry 2013;25:178-196. 